\title{
Optimum Control Parameters During Machining of LM13 Aluminum Alloy Under Dry Electrical Discharge Machining (EDM) With A Modified Tool Design
}

\author{
Pragadish NAGARAJAN ${ }^{1 *}$, Pradeep Kumar MURUGESAN ${ }^{2}$, Elango NATARAJAN ${ }^{3}$ \\ ${ }^{1}$ Faculty, Department of Mechanical Engineering, S.A. Engineering College, Chennai- 600077, India \\ ${ }^{2}$ Department of Mechanical Engineering, CEG, Anna University Chennai, Chennai-600 025, India \\ ${ }^{3}$ Faculty of Engineering, UCSI University, Kuala Lumpur, Malaysia \\ cross $^{\text {ref }}$ http://dx.doi.org/10.5755/j01.ms.25.3.20899
}

Received 06 June 2018; accepted 11 October 2018

\begin{abstract}
Dry Electrical Discharge Machining(EDM) is considered as a green manufacturing process in which the liquid dielectric medium is replaced by a high velocity gas, which results improved process stability. A special tool design is adopted to find the optimum control parameters during machining of LM13 Aluminum alloy under dry EDM mode. The drilled and slotted cylindrical copper rod is used as a tool. Discharge current $(I)$, voltage $(V)$, pressure $(P)$ and pulse on time $\left(T_{\mathrm{ON}}\right)$ are considered as varying input process parameters and duty factor and tool rotational speed are chosen at the fixed level. TaguchiL 27 orthogonal array is used to design the experiment and the experiments are conducted accordingly. The experimental results are analyzed using Grey Relational Analysis to find the optimal combination of the process parameters. Also, ANOVA test is conducted to ensure the conformity of the simulation results. Pulse on time is found as the most significant parameter which is followed by voltage. Furthermore, the parameters with the highest relational grade $(4 \mathrm{~A}, 200 \mu \mathrm{s}, 60 \mathrm{~V}$ and $1.5 \mathrm{kPa}$ ) are used in experiment to validate the simulation results. The simulation and experimental results have a good agreement with less than $0.5 \%$ error.

Keywords: EDM, dry EDM, ANOVA, optimization.
\end{abstract}

\section{INTRODUCTION}

In the conventional machining process, large cutting forces are involved, which result residual stresses in the work piece due to excess heat. Distortion is another parameter observed during machining of hard materials and complex shapes, which affects the life and quality of the work piece. Electrical Discharge Machining (EDM) being an unconventional manufacturing process is extensively applied in modern metal working industries. It is widely accepted in die making industries as the hardened steels can be easily cut with the EDM [1]. In EDM, the tool and the job does not have any direct contact, and thus greatly reduces the problems like chattering, vibration and mechanical stresses [2]. Some limitation of EDM process is recycling of the dielectric liquid and the generation of the toxic fumes due to the breakdown of the dielectric fluid during machining [3]. Dry EDM, a green manufacturing process uses gas as the dielectric medium which helps the users to overcome the limitation of conventional EDM process. The gas with high velocity is supplied into the discharge gap through the tubular tool that rotates at high speed. This reduces the arcs between the electrode and the work piece, flushes the debris particles more effectively and hence improves the process stability $[4,5,6]$. But the tubular tools do not support drilling of blind holes. The corresponding region in the work piece is not removed due to the absence of material at the centre of the tool that leads to the formation of a central core. This issue was overcome by some researchers by making some modifications in the solid rod [7].
A $10 \mathrm{~mm}$ diameter cylindrical copper rod with three uniformly distributed $2 \mathrm{~mm}$ diameter holes on its face was used to drill the holes in SS316 L [7]. Pragadish et al. [8] conducted the dry EDM machining on AISI D2 tool steel using a tool with specially modified design and observed increased material removal rate (MRR) and surface roughness (SR) as levels of current increases. Zhang et al. [9] provided ultrasonic vibration to the work piece and observed the improvement in MRR. Govindan et al. [6] used slotted electrode as the tool and observed enhanced values of MRR and reduction in tool wear. In spite of many advantages, comparatively low MRR has been reported in dry EDM due to low density of the gaseous dielectric. However, the usage of oxygen as the dielectric medium resulted better MRR in the dry EDM process [10].

Lin et al. [11] found the best combination of parameters using grey relational analysis (GRA) to improve the performance characteristics of the EDM process. Vinoth et al. [12] found the optimal parameters for cryogenic cooled EDM process using GRA. Kumar et al. [13] optimized the process parameters for machining of aluminum boron carbide, and reported current and pulse on time are the most significant parameters affecting MRR. Shen et al. [14] conducted experiments on Ti6Al4V titanium alloy using a novel high speed dry electrical discharge milling and observed the current as the main affecting factor on MRR and power consumption. However, only a few attempts have been made in optimizing the process parameters in dry EDM.

${ }^{*}$ Correspondin gauthor. Tel.: +091-9962567869

E-mail address: drpragadish@saec.ac.in (P. Nagarajan) 
This research attempts to minimize surface roughness and maximize material removal rate during the machining of LM13 Aluminum alloy through dry EDM process using a modified tool design. Taguchi L27 orthogonal array was used to design the experiments and Gray Rational Analysis (GRA) was used to optimize the parameters.

\section{EXPERIMENTAL DETAILS}

Electronica Xpert 1 type CNC Die sinking EDM machine was used to conduct the experiments. The three axes movement of the machine tool was achieved with the help of a DC servo system. Tool rotation was achieved using a special attachment as shown in the Fig. 1.LM13 aluminum alloy with the dimensions of $30 \mathrm{~mm} \times 30 \mathrm{~mm} \times 10 \mathrm{~mm}$ as shown in Fig. 2 was the material chosen. Oxygen gas and copper electrode were selected for the experiments as proved to be the best combination [15]. Oxygen gas with $99.99 \%$ purity was used as the dielectric medium and copper rod with $0.43 \%$ impurities was used as the tool.

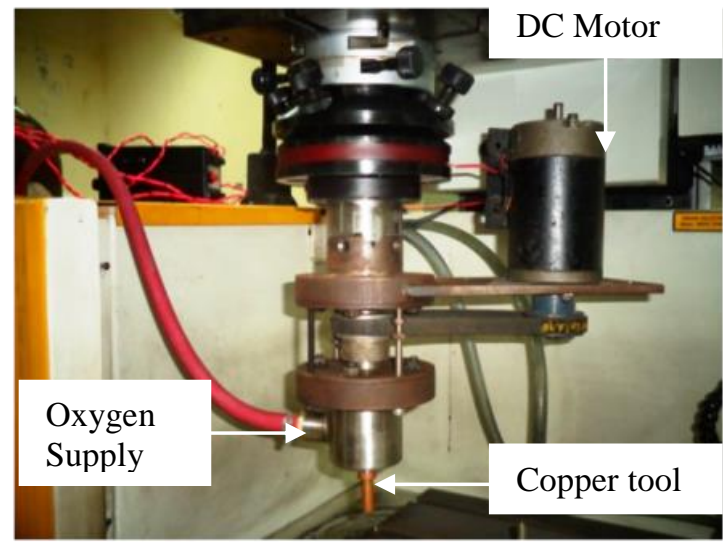

Fig. 1. Special attachment for conducting experiments in dry EDM process

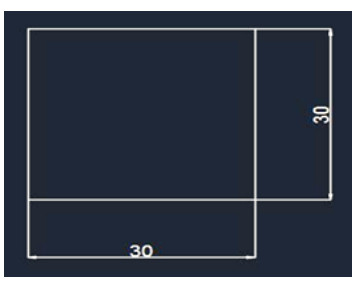

a

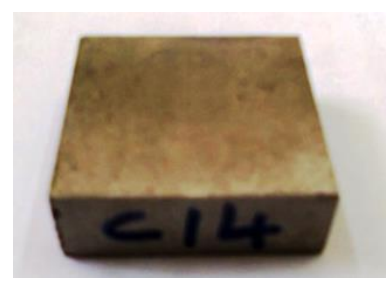

b
Fig. 2. a-CAD model of LM13 aluminum alloy work piece with dimension; $b$-LM13 aluminum alloy work piece

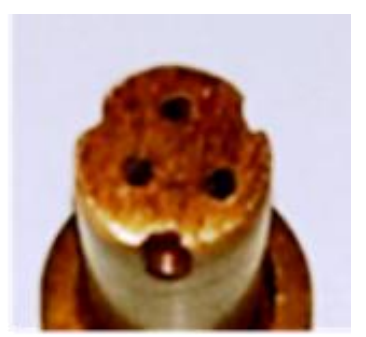

Fig. 3. Modified tool design

Pragadish et al. [8] proposed a special tool design for drilling holes in AISI D2 tool steel. $2 \mathrm{~mm}$ diameter hole was drilled on the base of the tool at three pitch circles of diameter $5 \mathrm{~mm}, 6 \mathrm{~mm}$ and $7 \mathrm{~mm}$. The holes were drilled along $180^{\circ}$ on the face of the tool. $2 \mathrm{~mm} \times 2 \mathrm{~mm}$ rectangular slots were cut on the alternate $180^{\circ}$, along the circumference. Initially this tool design was adopted in the current research, but encountered short circuiting problem during the machining due to ineffective flushing of the debris particles. The MRR was found more in LM13 aluminum alloy compared to AISI D2 tool steel and the slot length was found insufficient to enable the debris to flow out. The modified tool was designed with slot of $3 \mathrm{~mm}$ length as shown in Fig. 3 and the orthographic view is shown in the Fig. 4.

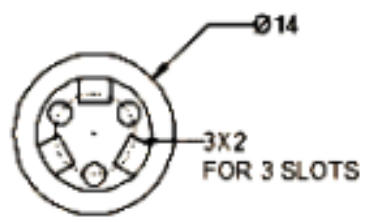

\section{BOTTOM VIEW}
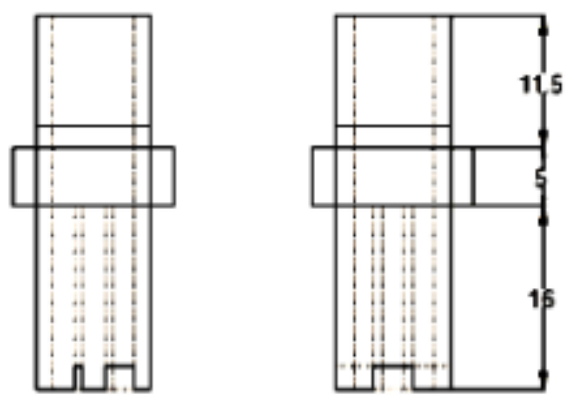

SIDE VIEW

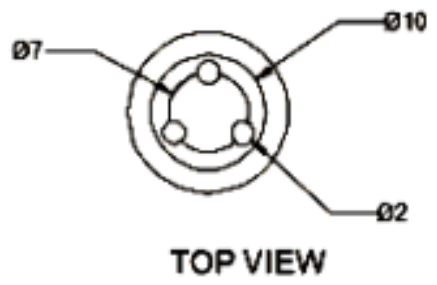

Fig. 4. Orthographic views of modified tool electrode design for machining LM13 aluminium alloy

The discharge current, voltage, gas pressure and pulse on time, at different levels were selected as the input parameters. Tool rotational speed and duty factor were set fixed as shown in Table 1.

Table 1. Input parameters and their values

\begin{tabular}{|l|c|c|c|}
\hline Factors & Level 1 & Level 2 & Level 3 \\
\hline Current, A & 4 & 6 & 8 \\
\hline Pulse on time, $\mu$ s & 100 & 150 & 200 \\
\hline Gap voltage, V & 50 & 60 & 70 \\
\hline Pressure, $\mathrm{kPa}$ & 1.5 & 2.0 & 2.5 \\
\hline Speed, rpm & 1500 & \multicolumn{3}{|l}{} \\
\hline Duty factor & 32 &
\end{tabular}

The effects of many parameters can be easily assessed with minimum number of experiments using Taguchi method, and it is an effective tool to optimize the problems involving single response variable [12]. Taguchi L27 
orthogonal array was designed and experiments were conducted accordingly. The input control variables and results of the experiments are given in Table 2. Each experiment was conducted for a constant period of three minutes and corresponding response variables were measured immediately after the experiments.

Grey Relation Analysis(GRA) is a multi-attribute decision making (MADM) methodology, which serves good in multi response variables. In 1982, Deng proposed Grey theory, which can effectively and precisely handle both the unclear problems and incomplete information. It can solve the problems with complicated interrelationships between multiple factors and variables. The system with complete information is represented as white and lack of information as black. The system with either incomplete or uncertain information is termed as Grey system. The term Grey relation represents the relation with incomplete information. The influence of various parameters and their relation in unclear situations can be established using Grey relation analysis [16-19].

The first step in GRA is Grey relational generation (GRG) in which the experimental data are normalized in the range between 0 and 1 . The overall performance of the optimization depends on the computation of GRG. Eq. 1 is applied to normalize the scattered data for larger the better performance characteristics and Eq. 2 is applied to normalize the scattered data for smaller the better performance characteristics.

$x_{i j}=\frac{y_{i j}-\min \left(y_{i j}, i=1,2, \ldots, n\right)}{\max \left(y_{i j}, i=1,2, \ldots, n\right)-\min \left(y_{i j}, i=1,2, \ldots, n\right)}$, $x_{i j}=\frac{\max \left(y_{i j}, i=1,2, \ldots, n\right)-y_{i j}}{\max \left(y_{i j}, i=1,2, \ldots, n\right)-\min \left(y_{i j}, i=1,2, \ldots, n\right)}$,

where $y_{i j}$ is the $i^{\text {th }}$ experimental result in the $j^{\text {th }}$ experiment.

The second step in the GRA is to find the grey relational coefficient (GRC) as;

$\gamma\left(x_{0 j}, x_{i j}\right)=\frac{\Delta_{\min }+\tau \Delta_{\max }}{\Delta_{i j}+\tau \Delta_{\max }}$,

where, $j=1,2, \ldots, n ; k=1,2, \ldots, m(n$ is the number of experiments and $m$ is the number of responses) and $\Delta_{0 \mathrm{j}}(\mathrm{k})$ is the absolute difference between the reference sequence $\left(x_{0}(\mathrm{k})\right)$ and the specific comparison sequence $\left(x_{\mathrm{i}}(\mathrm{k})\right)$ as given in $\mathrm{Eq} 4$.

$\Delta_{i j}=\left\|x_{0 j}-x_{i j}\right\|$

$\Delta_{\text {min }}=\operatorname{Min}\left(\Delta_{i j}, i=1,2, \ldots, n ; j=1,2, \ldots, m\right)$,

is the smallest value of $x_{\mathrm{ij}}$,

$\Delta_{\text {max }}=\operatorname{Max}\left(\Delta_{i j}, i=1,2, \ldots, n ; j=1,2, \ldots, m\right)$

is the largest value of $x_{\mathrm{ij}}$ and $\tau$ represents the distinguished coefficient ranging from 0 to 1 . Generally, it is taken as 0.5 .

The grey relational grade is calculated as;

$\overline{\gamma_{l}}=\frac{1}{j} \sum_{i=1}^{j} \gamma_{i j}$

where, $\bar{Y}_{t}$ is the grey relational grade for the $i^{\text {th }}$ experiment and $j$ is the number of responses.

Table 2. Input control variables and experimental results

\begin{tabular}{|c|c|c|c|c|c|c|}
\hline \multirow[b]{2}{*}{ S. No } & \multicolumn{4}{|c|}{ Control variables } & \multicolumn{2}{|c|}{ Response variables in dry EDM } \\
\hline & $\begin{array}{c}\text { Current } \\
I, \text { A }\end{array}$ & $\begin{array}{c}\text { Pulse on time } \\
T_{\mathrm{ON}}, \mu \mathrm{s}\end{array}$ & $\begin{array}{c}\text { Gap voltage } \\
V, \mathrm{~V}\end{array}$ & $\begin{array}{c}\text { Pressure } \\
P, \mathrm{kPa}\end{array}$ & $M R R, \mathrm{mgm} / \mathrm{minute}$ & $S R, \mu \mathrm{m}$ \\
\hline 1 & 4 & 100 & 50 & 1.5 & 7.87 & 7.21 \\
\hline 2 & 4 & 100 & 60 & 2 & 7.20 & 8.29 \\
\hline 3 & 4 & 100 & 70 & 2.5 & 7.60 & 9.70 \\
\hline 4 & 4 & 150 & 50 & 2 & 9.97 & 7.82 \\
\hline 5 & 4 & 150 & 60 & 2.5 & 11.30 & 8.18 \\
\hline 6 & 4 & 150 & 70 & 1.5 & 10.63 & 8.30 \\
\hline 7 & 4 & 200 & 50 & 2.5 & 10.50 & 9.83 \\
\hline 8 & 4 & 200 & 60 & 1.5 & 19.77 & 8.11 \\
\hline 9 & 4 & 200 & 70 & 2 & 11.50 & 7.00 \\
\hline 10 & 6 & 100 & 50 & 2 & 13.37 & 11.23 \\
\hline 11 & 6 & 100 & 60 & 2.5 & 10.90 & 10.22 \\
\hline 12 & 6 & 100 & 70 & 1.5 & 8.73 & 11.19 \\
\hline 13 & 6 & 150 & 50 & 2.5 & 10.87 & 11.66 \\
\hline 14 & 6 & 150 & 60 & 1.5 & 16.70 & 10.04 \\
\hline 15 & 6 & 150 & 70 & 2 & 11.93 & 10.55 \\
\hline 16 & 6 & 200 & 50 & 1.5 & 17.20 & 10.38 \\
\hline 17 & 6 & 200 & 60 & 2 & 19.83 & 9.35 \\
\hline 18 & 6 & 200 & 70 & 2.5 & 22.90 & 11.18 \\
\hline 19 & 8 & 100 & 50 & 2.5 & 12.80 & 10.72 \\
\hline 20 & 8 & 100 & 60 & 1.5 & 11.67 & 9.78 \\
\hline 21 & 8 & 100 & 70 & 2 & 10.23 & 11.38 \\
\hline 22 & 8 & 150 & 50 & 1.5 & 16.40 & 10.87 \\
\hline 23 & 8 & 150 & 60 & 2 & 12.00 & 10.35 \\
\hline 24 & 8 & 150 & 70 & 2.5 & 19.40 & 11.52 \\
\hline 25 & 8 & 200 & 50 & 2 & 16.37 & 12.38 \\
\hline 26 & 8 & 200 & 60 & 2.5 & 20.43 & 10.12 \\
\hline 27 & 8 & 200 & 70 & 1.5 & 21.60 & 12.22 \\
\hline
\end{tabular}


The experimental parameters corresponding to the highest grey relation grade is close to the optical combination to yield the better results.

In the next step, the quality characteristics are predicted and verified using the optimal level of design parameters. The predicted grey relational grade can be calculated as;

$\gamma_{\text {predicted }}=\gamma_{m}+\sum_{i=1}^{q}\left(\bar{\gamma}_{l}-\gamma_{m}\right)$,

where $Y_{m}$ refers to mean grey relational grade, $\bar{Y}_{t}$ refers to grey relational grade at the optimum level and q refers to the number of process parameters that influences the responses.

Larger the better characteristics was applied for MRR, and smaller the better characteristics was applied for SR. The GRC and grey relational grade were calculated using the Eq. 1-Eq. 6. The distinguishing factor $\tau$ was chosen to be 0.5 , as both responses are given equal weightage.

Analysis of Variance (ANOVA) is used to find the influence of individual input parameters on the multi performance characteristics and the process parameters that are significant over the responses. The influence of optimal parameters in machining of metals or composites can be investigated through ANOVA [20-22]. The effect of the individual factors over the response was analyzed in ANOVA using grey relation grades as the response at 95\%confidence level.

\section{RESULTS AND DISCUSSION}

Table 3 shows the values of the grey relational coefficient, the grey relational grade and the ranking of each experiment. It is observed that $17^{\text {th }}$ experiment has the highest grade among 27 experiments, which regarded to be the optimal value to acquire the best multi response characteristics of low SR and high MRR.

The average grey relational grade for each input parameter and the influential order of the parameters on the response is shown in the Table 4 . It is observed that, the levels A1 B3 C2 D1 have the highest grey relational grade for the factors $\mathrm{A}, \mathrm{B}, \mathrm{C}$ and $\mathrm{D}$ respectively. The values corresponding to the highest grey relational grade for the input parameters are $4 \mathrm{~A}, 200 \mu \mathrm{s}, 60 \mathrm{~V}$ and $1.5 \mathrm{kPa}$. Higher values of current may produce more heat which can easily erode more aluminium particles compared to the silicon particles which will degrade the surface finish, and also it requires more time for efficient melting and removal of work piece.

Table 3. Grey relational coefficient, grey relational grade and rank order

\begin{tabular}{|c|c|c|c|c|}
\hline \multirow[b]{2}{*}{ S. No } & \multicolumn{2}{|c|}{ Grey relational coefficient } & \multirow{2}{*}{$\begin{array}{l}\text { Grey } \\
\text { relation } \\
\text { grade }\end{array}$} & \multirow{2}{*}{$\begin{array}{c}\text { Ranking } \\
\text { order }\end{array}$} \\
\hline & $\begin{array}{c}\text { Material } \\
\text { removal rate }\end{array}$ & $\begin{array}{c}\text { Surface } \\
\text { roughness }\end{array}$ & & \\
\hline 1 & 0.04 & 0.96 & 0.5020 & 1 \\
\hline 2 & 0.00 & 0.76 & 0.3802 & 2 \\
\hline 3 & 0.03 & 0.50 & 0.2614 & 3 \\
\hline 4 & 0.18 & 0.85 & 0.5116 & 4 \\
\hline 5 & 0.26 & 0.78 & 0.5213 & 5 \\
\hline 6 & 0.22 & 0.76 & 0.4881 & 6 \\
\hline 7 & 0.21 & 0.47 & 0.3425 & 7 \\
\hline 8 & 0.80 & 0.79 & 0.7969 & 8 \\
\hline 9 & 0.27 & 1.00 & 0.6374 & 9 \\
\hline 10 & 0.39 & 0.21 & 0.3032 & 10 \\
\hline 11 & 0.24 & 0.40 & 0.3189 & 11 \\
\hline 12 & 0.10 & 0.22 & 0.1591 & 12 \\
\hline 13 & 0.23 & 0.13 & 0.1833 & 13 \\
\hline 14 & 0.61 & 0.43 & 0.5198 & 14 \\
\hline 15 & 0.30 & 0.34 & 0.3213 & 15 \\
\hline 16 & 0.64 & 0.37 & 0.5042 & 16 \\
\hline 17 & 0.80 & 0.56 & 0.6842 & 17 \\
\hline 18 & 1.00 & 0.22 & 0.6115 & 18 \\
\hline 19 & 0.36 & 0.31 & 0.3330 & 19 \\
\hline 20 & 0.28 & 0.48 & 0.3837 & 20 \\
\hline 21 & 0.19 & 0.19 & 0.1891 & 21 \\
\hline 22 & 0.59 & 0.28 & 0.4335 & 22 \\
\hline 23 & 0.31 & 0.38 & 0.3413 & 23 \\
\hline 24 & 0.78 & 0.16 & 0.4683 & 24 \\
\hline 25 & 0.58 & 0.00 & 0.2916 & 25 \\
\hline 26 & 0.84 & 0.42 & 0.6315 & 26 \\
\hline 27 & 0.92 & 0.03 & 0.4734 & 27 \\
\hline
\end{tabular}

Low voltage may lead to decrease in the inter electrode distance and may cause frequent arcing, and higher voltage levels may lead to increase in the distance leading to improper machining. $1.5 \mathrm{kPa}$ pressure is sufficient for flushing of debris particles and further increase in pressure has no significant effect, and this is also evident from the grey relational grade ranking shown in the Table 4. It is also observed that, the pulse on time (TON) is the most influencing factor followed by voltage (V), current (A) and the pressure (P) for obtaining the bestmultiresponsecharacteristics.

Table 4. Grey relational grade

\begin{tabular}{|c|c|c|c|c|c|c|}
\hline Symbol & Parameter & Level 1 & Level 2 & Level 3 & Delta & Rank \\
\hline A & Current, A & $\mathbf{0 . 4 9 3 5}$ & 0.4006 & 0.3739 & 0.1196 & 2 \\
\hline B & Pulse ontime, TON & 0.3145 & 0.4209 & $\mathbf{0 . 5 5 2 6}$ & 0.2381 & 1 \\
\hline C & Voltage, V & 0.3983 & 0.4011 & $\mathbf{0 . 5 0 8 6}$ & 0.1103 & 3 \\
\hline D & Pressure, P & $\mathbf{0 . 4 7 3 4}$ & 0.4066 & 0.4080 & 0.0654 & 4 \\
\hline
\end{tabular}

Table 5. Results of confirmation experiment

\begin{tabular}{|c|c|c|c|c|c|c|}
\hline \multirow[b]{2}{*}{ Experiment } & \multicolumn{4}{|c|}{ Machiningparameters } & \multicolumn{2}{|c|}{ Response } \\
\hline & $\begin{array}{c}\text { Current, } \\
\text { A }\end{array}$ & Pulse ontime, ToN & Voltage, V & Pressure, $\mathrm{P}$ & $\begin{array}{c}\mathrm{MRR}, \\
\mathrm{mgm} / \mathrm{min}\end{array}$ & $\mathrm{SR}, \mu \mathrm{m}$ \\
\hline Initialexperimentvalues & 4 & 200 & 60 & 1.5 & 19.77 & 8.11 \\
\hline Confirmationexperimentvalues & 4 & 200 & 60 & 1.5 & 19.80 & 8.08 \\
\hline \multicolumn{5}{|c|}{ Percentage error } & $0.15 \%$ & $0.37 \%$ \\
\hline
\end{tabular}


Table 6. ANOVA for grey relational grade

\begin{tabular}{|c|c|c|c|c|c|c|}
\hline Source & DOF & Sumofsquare & Meansquare & F & P & \% ofcontribution \\
\hline I & 2 & 0.02240 & 0.01120 & 0.74 & 0.491 & 8.71 \\
\hline TON & 2 & 0.18501 & 0.09250 & 6.12 & 0.009 & $71.91^{* *}$ \\
\hline V & 2 & 0.04422 & 0.02211 & 1.46 & 0.258 & $17.19^{*}$ \\
\hline P & 2 & 0.00567 & 0.00283 & 0.19 & 0.831 & 2.20 \\
\hline ERROR & 18 & 0.27218 & 0.01512 & & & \\
\hline TOTAL & 26 & 0.52947 & & & & \\
\hline $\begin{array}{l}* * \text { Most influencing factor } \\
* \text { Influencing factor }\end{array}$
\end{tabular}

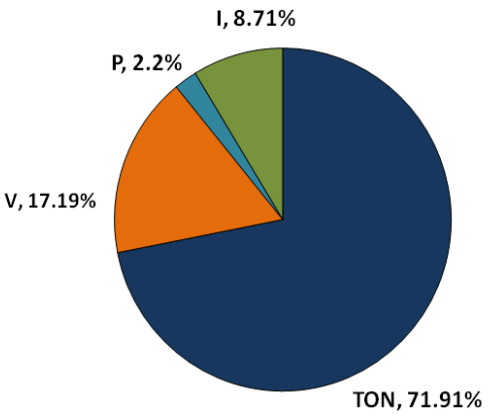

Fig. 5. Percentage contribution of process parameters

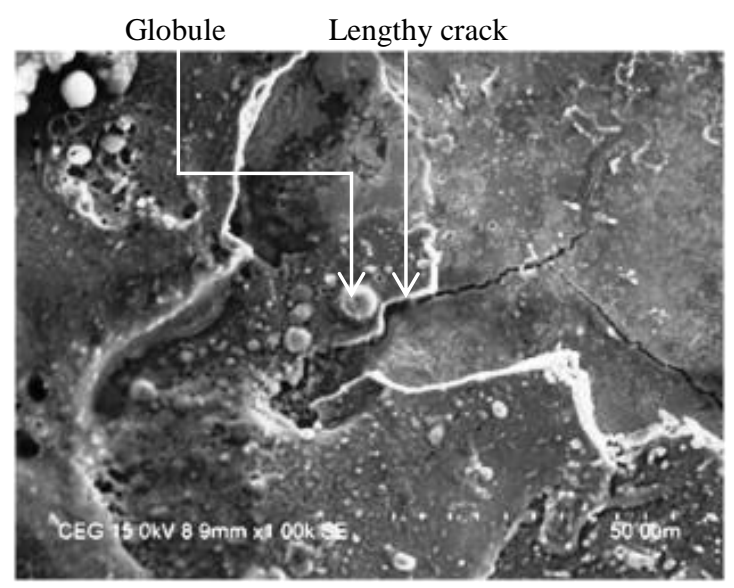

a

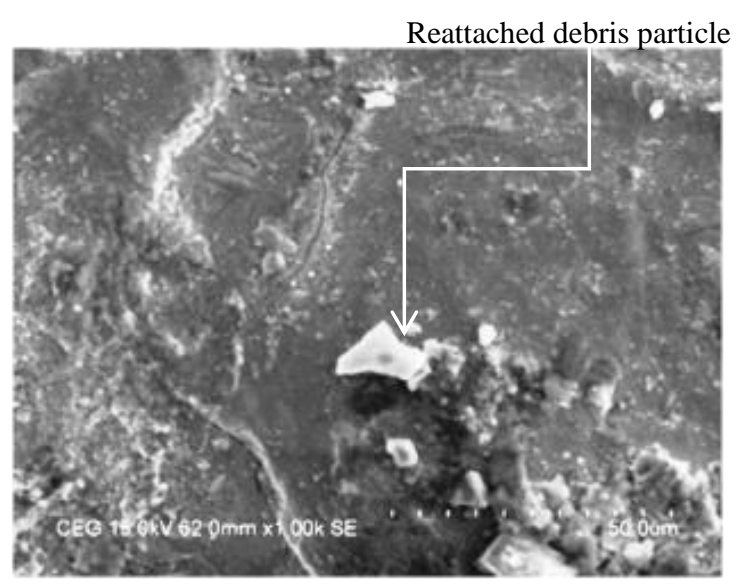

b

Fig. 6. SEM image of the surfaces machined with optimal parameters under: $\mathrm{a}-\mathrm{CEDM}$ process; $\mathrm{b}-$ dry EDM process
Furthermore, the parameters with the highest relational grade viz. $4 \mathrm{~A}, 200 \mu \mathrm{s}, 60 \mathrm{~V}$ and $1.5 \mathrm{kPa}$ were used and experiment was conducted to validate the simulation results.

The error of $0.3 \%$ on MRR and $0.37 \%$ on SR were observed between the initial and confirmation results. Table 5 shows the initial experimental values and validated experimental values.

The effect of the individual factors over the response is shown in Table 6. The significance of the parameter under investigation is based on the $F$ value. If the calculated value of the F-ratio is higher than the tabulated F value, the factor is considered significant. From the results, it is found that pulse on time (TON) is the only factor significant at $95 \%$ confidence interval. As shown in Fig. 4, the pulse on time $(71.91 \%)$ is the most influential factor followed by the voltage $(17.19 \%)$, the current $(8.71 \%)$ and, the pressure $(2.2 \%)$.

The SEM images of the surfaces machine under CEDM process and dry EDM process for the optimal parameters is shown in the Fig. 5. The length cracks are more visible in the surface machine under CEDM process. Thepresenceof globules is also noticed. The surface machine under dry EDM process does not reveal any cracks and shows the better surface finish. But, the reattachment of debris particles is also observed, which is due to inefficient flushing of the debris particles.

\section{CONCLUSIONS}

Experiments were conducted on LM13 aluminum alloy under the dry EDM process based on $\mathrm{L}_{27}$ orthogonal array. The optimal combination of the process parameters was found using the grey relational analysis and the following conclusions were arrived at.

1. From GRA, the $8^{\text {th }}$ experiment was found to have the optimal parameters $A_{1}=4 A, B_{3}=200 \mu \mathrm{s}, C_{2}=60 \mathrm{~V}$ and $\mathrm{D}_{1}=1.5 \mathrm{kPa}$ with the highest grey relational grade of 0.7969 .

2. The results of the confirmatory experiments are very close to the results achieved in the initial experiments.

3. From the ANOVA test, it was observed that the pulse on time $71.91 \%$ is the most influential factor followed by the voltage of $17.19 \%$.

No cracks were observed from the SEM image of the surface machined under the dry EDM process with the optimal parameters. But the reattachment of debris particles is observed. 


\section{REFERENCES}

1. CheHaron, C.H., Md Deros, B., Ginting, A., Fauziah, M. Investigation on the Influence of Machining Parameters When Machining Tool Steel Using EDM Journal of Materials Processing Technology $116(1)$ 2001: pp. $84-87$.

https://doi.org/10.1016/S0924-0136(01)00846-9

2. Abbas, N.M., Darius, G., Solomon Bahari, M.F. A Review on Current Research Trends in Electrical Discharge Machining (EDM) International Journal of Machine Tools and Manufacture 47 (7-8) 2007: pp. 1214-1228. https://doi.org/10.1016/j.ijmachtools.2006.08.026

3. Abbas, N.M., Yusoff, N., Mahmod, R. Electrical Discharge Machining (EDM): Practices in Malaysian Industries and Possible Change Towards Green Manufacturing Procedia Engineering 41 2012: pp. 1684-1688. https://doi.org/10.1016/j.proeng.2012.07.368

4. Saha, S.K., Choudhury, S.K. Experimental Investigation and Empirical Modeling of the Dry Electric Discharge Machining Process International Journal of Machine Tools and Manufacture 49 (3-4) 2009: pp. 297-308.

https://doi.org/10.1016/j.ijmachtools.2008.10.012

5. Kunieda, M., Yoshida, M. Electrical Discharge Machining in Gas CIRP Annals 46 (1) 1997: pp. 143-146. https://doi.org/10.1016/S0007-8506(07)60794-X

6. Govindan, P., Joshi, S.S. Investigations into Performance of Dry EDM Using Slotted Electrodes International Journal of Precision Engineering and Manufacturing 12 (6) 2011: pp. $957-963$. https://doi.org/10.1007/s12541-011-0128-2

7. Murickan, R.T., $\quad$ Lakshmipathy, J., Kuppan, P. Experimental Investigation of Dry Electrical Discharge Machining on SS 316L International Journal of Latest Trends in Engineering and Technology 2(3) 2013: pp. 100-107.

8. Pragadish, N., Pradeep, K.M., Surface Characteristics Analysis of Dry EDMed AISI D2 steel Using Modified Tool Design Journal of Mechanical Science and Technology 29 (4) 2015: pp. $1737-1743$

https://doi.org/10.1007/s12206-015-0347-z

9. Zhang, Q.H., Du, R., Zhang, J.H., Zhang, Q.B. An Investigation of Ultrasonic-Assisted Electrical Discharge Machining in Gas International Journal of Machine Tools and Manufacture $46(12-13)$ 2006: pp. 1582-1588. https://doi.org/10.1016/j.ijmachtools.2005.09.023

10. Shabgard, M.R., Gholipoor, A., Baseri, H. A Review on Recent Developments in Machining Methods Based on Electrical Discharge Phenomena The International Journal of Advanced Manufacturing Technology 87 (5-8) 2016: pp. $2081-2097$. https://doi.org/10.1007/s00170-016-8554-Z

11. Lin, J.L., Lin, C.L. The Use of the Orthogonal Array with Grey Relational Analysis to Optimize the Electrical Discharge Machining Process with Multiple Performance
Characteristics International Journal of Machine Tools and Manufacture 42 (2) 2002: pp. 237-244.

https://doi.org/10.1016/S0890-6955(01)00107-9

12. VinothKumar, S., Pradeep, K.M. Optimization of Cryogenic Cooled EDM Process Parameters Using Grey Relational Analysis Journal of Mechanical Science and Technology 28 (9) 2014: pp. 3777-3784. https://doi.org/10.1007/s12206-014-0840-9

13. Kumar, P., Parkash, R. Experimental Investigation and Optimization of EDM Process Parameters for Machining of Aluminum Boron carbide (Al-B4C) Composite Machining Science and Technology 20 (2) 2016: pp. 330-348. http://dx.doi.org/10.1080/10910344.2016.1168931

14. Shen, Y., Liu, Y., Dong, H., Lin, K.Z., Zhang, X., Zheng, C., Ji, R. Parameters Optimization for Sustainable Machining of Ti6Al4Vusing a Novel High-Speed Dry Electrical Discharge Milling The International Journal of Advanced Manufacturing Technology 90 (9-12) 2017: pp. $2733-2740$. http://dx.doi.org/10.1007/s00170-016-9600-6

15. Tao, J.,Shih, A.J., Ni, J. Experimental Study of the Dry and Near-Dry Electrical Discharge Milling Processes Journal of Manufacturing Science and Engineering 130 (1) 2008: pp.011002-1-9. http://dx.doi.org/10.1115/1.2784276

16. Chakravorty, R., Gauri, S.K., $\quad$ Chakraborty, S. Optimization of Correlated Responses of EDM Process Materials and Manufacturing Process $27(3)$ 2012: pp. $337-347$ http://dx.doi.org/10.1080/10426914.2011.577875

17. Moran, J., Granada, E., Mı'guez, J.L., Porteiro, J. Useof Grey Relational Analysis to Assess and Optimize Small Biomass Boilers Fuel Processing Technology 87 (2) 2006: pp. $123-127$. https://doi.org/10.1016/j.fuproc.2005.08.008

18. Caydas, U., Hascalik, A. Useof the Grey Relational Analysis to Determine Optimum Laser Cutting Parameters with MultiPerformance Characteristics Optics and Laser Technology 40 (7) 2008: pp. $987-994$. https://doi.org/10.1016/j.optlastec.2008.01.004

19. Hasani, H., Tabatabaei, S.A., Amiri, G. Grey Relational Analysis to Determine the Optimum Process Parameters for Open-End Spinning Yarns Journal of Engineered Fibers and Fabrics 7 (2) 2012: pp. 81-86.

20. Viswanathan, R., Ramesh, S., Elango, N., Kamesh, K.D. Temperature Measurement and Optimization in Machining Magnesium Alloy Using RSM and Annova Pertanika Journal of Science and Technology 25 (1) 2017: pp. 255-262.

21. Suresh, P., Venkatesan, R., Sekar, T.P., Elangon, N., Sathiyamoorthy, V. Optimization of Intervening Variables in Microedm of SS 316L Using a Genetic Algorithm and Response-Surface Methodology Journal of Mechanical Engineering 60 s(10) 2015: pp. 656-664. 